\title{
EFECTO DE LOS AGENTES EDUCADORES Y MODALIDAD DE INGRESO EN EL RENDIMIENTO ACADÉMICO DE LOS ESTUDIANTES DE LA UNCP
}

\section{EFFECT OF EDUCATOR AGENTS AND MEMBERSHIP METHOD IN THE ACADEMIC PERFORMANCE OF UNCP STUDENTS}

\author{
Miguel Ángel Huaringa Sánchez, María Elena Aliaga Guerra \\ Instituto de investigación de la Facultad de Economía
}

\section{RESUMEN}

El presente trabajo de investigación, se formuló con el propósito de determinar el efecto de los agentes educadores (familia, colegio de procedencia) y de la modalidad de ingreso en el rendimiento académico de los estudiantes de la UNCP, teniendo en consideración que la formación de nuestros estudiantes, no solo se da como resultado de lo que acontece en las aulas, sino que también influyen otros factores relacionados a los aspectos socioeconómicos. Para el efecto se utilizó un diseño de corte transversal, aplicando una encuesta para levantar la información de los aspectos socioeconómicos (agentes educadores) de los estudiantes. Esta data se cruzó con la información proporcionada por la Oficina de Registros Académicos de la UNCP, respecto a los promedios ponderados del semestre y acumulado de los estudiantes. Para la diferencia de medias se utilizó el análisis de varianza y la prueba de muestras independientes. En el análisis determinamos que solo las variables modalidad de ingreso, dependencia económica del estudiante y lugar donde toman sus alimentos, influyen en el rendimiento académico de los estudiantes. Las variables sexo de los estudiantes, semestre académico, grado de instrucción de los padres, ocupación de los padres, lugar de origen del estudiante, tipo de colegio donde estudió, y academia pre-universitaria donde se preparó para el ingreso, no influyen significativamente en el rendimiento académico de los estudiantes.

Palabras clave: agentes educadores, rendimiento académico, modalidad de ingreso.

\begin{abstract}
This investigation work, was formulated with the purpose of determinate the effect of the education agents (family or precedent school) and the apply mode, on academic performance of UNCP students, having consideration that formation of our students is not only result of what happens in the classroom but also other factors related whit socioeconomic aspects have influences too. For the effect we used a crosssection design, using a survey for up the information of the socioeconomic aspects (education agents) of the students. This data was crossed with the information provided by the Office of Academic Records of the UNCP, about the weighted average of the semester and cumulative grade point average of students. For the mean difference was used analysis of variance and independent samples test. The analysis determined that only the variables: student's type of admission, financial dependence and place where they take their food, have influence on the academic performance of our students. The variables: students sex, academic semester, parents degree of instruction, parents occupation, original city of the students, type of school, and precollege academy studied, don't have important influence on the academic performance our students.
\end{abstract}

Key words: University teaching, evaluation, survey of student appreciation 


\section{INTRODUCCIÓN}

En la Universidad Nacional del Centro del Perú, como reflejo de las tendencias que exige un mundo globalizado, se ha afianzado el camino hacia el modelo curricular por competencias, en el cual se toma un especial empeño en el desarrollo de capacidades profesionales de los egresados, lo que implica hablar de un perfil del egresado y hacia èl van encaminados los esfuerzos de la institución.

Sin embargo, no solo se debe analizar el comportamiento de los factores internos de nuestra Casa Superior de Estudios, como son calidad de la enseñanza, infraestructura educativa, actualización de la currícula, etc., sino que, es necesario identificar además el perfil del ingresante, y más aún, conocer cuál es el efecto de los diferentes agentes educadores: (familia, colegio, lugar de procedencia, entre otros), en el rendimiento académico de los estudiantes de cada una de las facultades de la UNCP. Por otro lado, en muchas de nuestras unidades académicas, no se tiene bien en claro el perfil del ingresante, y lo que es más crítico, el Examen de Admisión que sirve para clasificar a los postulantes más aptos para seguir estudios universitarios, muchas veces no toma en cuenta los requerimientos del perfil del ingresante, resultado del cual, los niveles de deserción y repitencia estudiantil podrían ser más significativos. En consecuencia es urgente efectuar un estudio respecto del perfil de los ingresantes respecto de su rendimiento académico para tomar decisiones en política de Admisión.

La Comisión de Admisión de la UNCP, en su esfuerzo de estandarizar el sistema de ingreso a nuestra Casa Superior de Estudios, ha clasificado a todas las facultades en cinco áreas de especialización, las cuales son: I Ciencias de la Salud; II Ciencias Agrarias; III Ingenierías y Arquitectura; IV Ciencias Económicas, Administrativas y Contables; y V Ciencias Sociales. De igual manera las modalidades de Ingreso son: a) Primeros Puestos, b) Vía Centro Pre Universitario, c) Examen de Admisión (Examen Regular y Primera Selección), d) Deportistas Destacados, e) Hijos de Comuneros y f) Víctimas del terrorismo. Por tanto, las hipótesis de investigación fueron: Hipótesis general; El rendimiento académico de los estudiantes de las Facultades de Economía, Administración, Contabilidad y
Educación (Especialidad Ciencias Sociales) de la UNCP, Período 2005-2009, está influenciado por la acción de los agentes educadores (familia, colegio, procedencia) y de la modalidad de ingreso. Hipótesis Específicas a) Existe una relación positiva entre las características familiares y el rendimiento académico de los estudiantes de las facultades de Economía, Administración, Contabilidad y Educación (Especialidad Ciencias Sociales) de la UNCP.; b) Existen diferencias significativas en el rendimiento académico de los estudiantes de las facultades de Economía, Administración, Contabilidad y Educación (Especialidad Ciencias Sociales) de la UNCP, respecto de las diferentes modalidades de ingreso.

Nuestros objetivos planteados fueron: objetivo general; determinar el efecto de los agentes educadores (familia, colegio, procedencia) y de la modalidad de ingreso, en el rendimiento académico de los estudiantes de la UNCP. específicos: a. Analizar el efecto de los agentes educadores en el rendimiento académico de los estudiantes de la UNCP., b) Analizar el efecto de la modalidad de ingreso en el rendimiento académico de los estudiantes de la UNCP

\section{MATERIAL Y MÉTODOS}

El método de investigación utilizado fue el científico. Como métodos específicos, se utilizaron el deductivo-inductivo; el análisis y síntesis. La investigación fue de tipo explicativo y correlacional, pues trató de indagar si las características familiares y la modalidad de ingreso explican el rendimiento académico de los estudiantes de la UNCP.

El rendimiento académico está representado por el promedio ponderado en el semestre 2010-2; el promedio ponderado acumulado para el mismo periodo. Esta información se obtuvo de la oficina de asuntos académicos.

La población está constituida por el rendimiento académico de los estudiantes de la Universidad Nacional del Centro del Perú, período 2006-2010. Para el efecto se trabajó con una muestra de 90 estudiantes de economía, a quiénes se les aplicó una encuesta orientada a conocer las principales características socioeconómicas, familiares y modalidad de ingreso, las mismas que fueron 
cruzadas con su rendimiento académico.

Para efectuar la sistematización de los resultados obtenidos se hizo uso de la hoja de cálculo Excel 2007, del software estadístico SPSS y del software econométrico Eviews.

\section{RESULTADOS}

\section{EDAD DE LOS ENTREVISTADOS}

Los estudiantes tienen una edad entre 18 y 30 años con una alta incidencia de las edades 20 y 22 años. De ello se puede deducir que las edades de ingreso a la Universidad está entre los 16 y 18 años, que es un comportamiento muy generalizado en las universidades de nuestra región.

\section{DISTRIBUCIÓN POR SEXO DE LOS ENTREVISTADOS}

Una clara tendencia que se tiene en la Facultad de Economía es la mayor frecuencia de estudiantes del sexo femenino que se inclinan por seguir los estudios universitarios, tal como se muestra en el cuadro No. 01, donde se muestra que el $52.20 \%$ de los matriculados pertenecen al sexo femenino, mientras que el $47.8 \%$ de los estudiantes son varones.

Cuadro No. 01 Distribución de los estudiantes según sexo del entrevistado.

\begin{tabular}{lcccc}
\hline Sexo & Frecuencia & $\%$ & $\begin{array}{c}\text { Porcentaje } \\
\text { válido }\end{array}$ & $\begin{array}{c}\text { Porcentaje } \\
\text { acumulado }\end{array}$ \\
\hline Femenino & 47 & 52.2 & 52.2 & 52.2 \\
Masculino & 43 & 47.8 & 47.8 & 100.0 \\
Total & 90 & 100.0 & 100.0 & \\
\hline
\end{tabular}

\section{PROCEDENCIA DE LOS ESTUDIANTES}

El $70 \%$ de los estudiantes proceden de las provincias del valles del Mantaro, mientras que un $16,7 \%$ proviene de la región Huancavelica, siendo marginal la procedencia de otras regiones. Ello nos demuestra que nuestra universidad es preferida por estudiantes de la región y de estudiantes huancavelicanos, no teniendo una demanda de estudiantes de otras regiones.
Cuadro No. 02: Distribución de los estudiantes según región de origen.

\begin{tabular}{ccccc}
\hline Región & Frecuencia & Porcentaje & $\begin{array}{c}\text { Porcentaje } \\
\text { válido }\end{array}$ & $\begin{array}{c}\text { Porcentaje } \\
\text { acumulado }\end{array}$ \\
\hline Junín & 70 & 77.8 & 77.8 & 77.8 \\
Huancavelica & 15 & 16.7 & 16.7 & 94.4 \\
$\begin{array}{c}\text { Cerro de } \\
\text { Pasco }\end{array}$ & 2 & 2.2 & 2.2 & 96.7 \\
Cajamarca & 1 & 1.1 & 1.1 & 97.8 \\
Lima & 2 & 2.2 & 2.2 & 100.0 \\
Total & 90 & 100.0 & 100.0 & \\
\hline
\end{tabular}

\section{INSTITUCIÓN EDUCATIVA DE PROCEDENCIA}

Como se muestra líneas abajo, los estudiantes matriculados en la UNCP, provienen fundamentalmente de colegios públicos $(89 \%)$, mientras que los demás proceden de colegios privados (11\%)

Cuadro No. 03: Distribución de los estudiantes según tipo de I. E.

\begin{tabular}{cccccc}
\hline $\begin{array}{c}\text { Institución } \\
\text { Educativa }\end{array}$ & Frecuencia & $\%$ & $\begin{array}{c}\text { Porcenta } \\
\text { je válido }\end{array}$ & $\begin{array}{c}\text { Porcentaje } \\
\text { acumulado }\end{array}$ \\
\hline \multirow{4}{*}{ Válidos } & Pública & 80 & 88.9 & 88.9 & 88.9 \\
& Privada & 10 & 11.1 & 11.1 & 100.0 \\
& Total & 90 & 100.0 & 100.0 & \\
\hline
\end{tabular}

\section{MODALIDAD DE INGRESO DE LOS ESTUDIANTES}

Los estudiantes de la UNCP, logran su ingreso, por la modalidad de Examen de Admisión Regular $(67,8 \%)$, y por la modalidad de estudios en el Centro Pre Universitario de la Universidad $(23,3 \%)$, cobra especial importancia también la modalidad de ingreso por Primera Selección $(4,4 \%)$, primeros puestos, traslado externo y traslado interno.

Cuadro No. 04: Distribución de los estudiantes según modalidad de ingreso

\begin{tabular}{lcccc}
\hline $\begin{array}{l}\text { Modalidad de } \\
\text { Ingreso }\end{array}$ & Frecuencia & $\%$ & $\begin{array}{c}\text { Porcentaje } \\
\text { válido }\end{array}$ & $\begin{array}{c}\text { Porcentaje } \\
\text { acumulado }\end{array}$ \\
\hline $\begin{array}{l}\text { Examen } \\
\text { Admisión regular }\end{array}$ & 61 & 67.8 & 67.8 & 67.8 \\
$\begin{array}{l}\text { Cepre } \\
\text { Traslado externo }\end{array}$ & 21 & 23.3 & 23.3 & 91.1 \\
$\begin{array}{l}\text { Primera } \\
\text { Selección }\end{array}$ & 4 & 1.1 & 1.1 & 92.2 \\
$\begin{array}{l}\text { Traslado interno } \\
\text { Primeros }\end{array}$ & 1 & 4.4 & 4.4 & 96.7 \\
puestos & 2 & 1.1 & 1.1 & 97.8 \\
Total & 90 & 2.2 & 2.2 & 100.0 \\
\hline
\end{tabular}




\section{APOYO EN LA SELECCIÓN DE LA FACULTAD}

Se indagó en los estudiantes, si tuvieron alguna orientación de los padres, profesores, hermanos u otros familiares, en la selección de la facultad, encontrando que curiosamente solo el $17,8 \%$ de ellos tuvo un asesoramiento y/o sabía a que facultad presentarse de acuerdo a su vocación. Ello quiere decir, que generalmente, un error muy grande que se viene cometiendo, es la falta de orientación a los estudiantes de los últimos grados de estudio secundario para definir su, lo cual puede repercutir en el rendimiento académico de los estudiantes, y en el caso extremo el abandono de la carrera al no tener las expectativas seguras.

Cuadro No. 05: Seleccionó la carrera por Orientación Vocacional

\begin{tabular}{lcccc}
\hline $\begin{array}{c}\text { Por qué seleccionó } \\
\text { la carrera }\end{array}$ & Frecuencia & $\%$ & $\begin{array}{c}\text { Porcentaj } \\
\text { e válido }\end{array}$ & $\begin{array}{c}\text { Porcentaje } \\
\text { acumulado }\end{array}$ \\
\hline $\begin{array}{l}\text { Orientación } \\
\text { vocacional } \\
\text { otros motivos }\end{array}$ & 16 & 17.8 & 17.8 & 17.8 \\
Total & 74 & 82.2 & 82.2 & 100.0 \\
& 90 & 100.0 & 100.0 & \\
\hline
\end{tabular}

\section{DEPENDENCIA ECONÓMICA}

Los estudiantes de la UNCP, en las facultades estudiadas, tienen dependencia económica de sus progenitores, en un primer caso $55,6 \%$ de las familias son estables (padre y madre juntos), en un $14,4 \%$ dependen del padre solamente y en un $16 \%$ dependen de la madre únicamente. Un 11,1\% manifiestan ser independientes.

Cuadro No. 06: Dependencia económica de los estudiantes

\begin{tabular}{llcccc}
\hline Dependencia económica & $\begin{array}{c}\text { Frecue } \\
\text { ncia }\end{array}$ & $\%$ & $\begin{array}{c}\text { Porcentaje } \\
\text { válido }\end{array}$ & $\begin{array}{c}\text { Porcentaje } \\
\text { acumulado }\end{array}$ \\
\hline \multirow{4}{*}{ Válidos } & Papá & 13 & 14.4 & 14.4 & 14.4 \\
& Mamá & 15 & 16.7 & 16.7 & 31.1 \\
& Papá y mamá & 50 & 55.6 & 55.6 & 86.7 \\
& Hermanos & 1 & 1.1 & 1.1 & 87.8 \\
& Abuelos-tíos & 1 & 1.1 & 1.1 & 88.9 \\
& Independiente & 10 & 11.1 & 11.1 & 100.0 \\
\hline & Total & 90 & 100.0 & 100.0 & \\
\hline
\end{tabular}

\section{RENDIMIENTO ACADÉMICO DE LOS ESTUDIANTES}

El análisis del rendimiento académico de los estudiantes se efectúa a través del promedio ponderado semestral y promedio semestral acumulado, obtenido de la oficina de Asuntos Académicos de la Facultad. De igual manera, se utilizó la calificación del estudiante a través de su situación y condición que son parámetros utilizados por Asuntos Académicos para la calificación y selección de los estudiantes.

\section{Promedio Ponderado Semestral y Promedio Ponderado Acumulado}

El calificativo promedio ponderado por semestre en los estudiantes entrevistados fue de 10,80 con un desviación estándar de 1,544 y un coeficiente de variación del $14.3 \%$, que nos indica un comportamiento homogéneo de los datos.

De igual manera, se hizo un análisis de los promedios ponderados acumulados por los estudiantes encontrando una media de 11,51, una desviación típica de 1,41 y un coeficiente de variabilidad de $12.3 \%$ que también indica un comportamiento homogéneo de las calificaciones.

Promedio ponderado semestral de los estudiantes

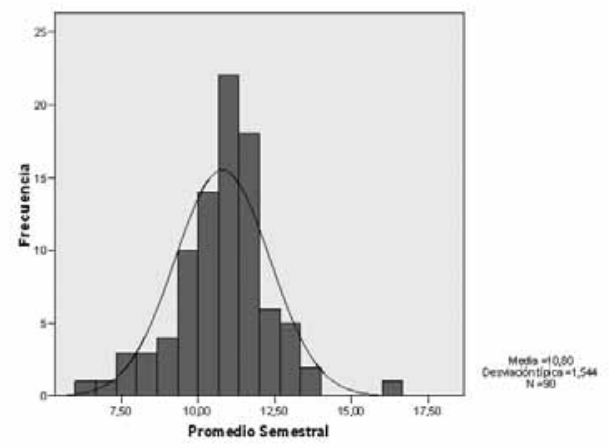

Promedio acumulado de los estudiantes 


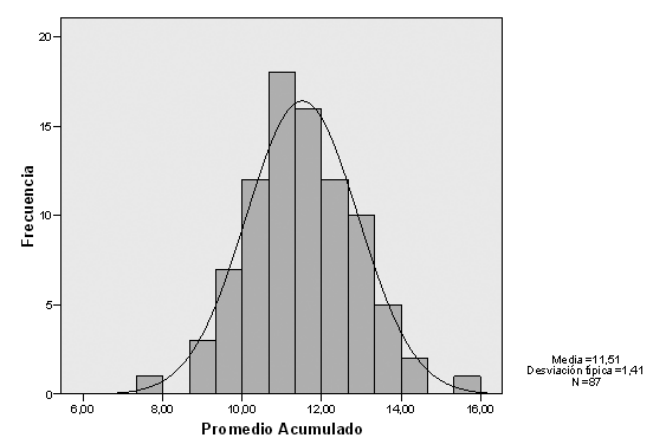

\section{Condición de los estudiantes}

Al efectuar el análisis de la condición de los estudiantes, se observa que un $30 \%$ de los estudiantes presenta bajo rendimiento académico (suspendidos o pagantes permanentes), mientras que un $70 \%$ tienen un comportamiento normal. Se incluye un estudiante que es hijo de trabajadores universitarios.

Cuadro No. 07: Distribución de los estudiantes según su condición

\begin{tabular}{lcccc}
\hline $\begin{array}{c}\text { Condición de } \\
\text { los } \\
\text { estudiantes }\end{array}$ & Frecuencia & Porcentaje & $\begin{array}{c}\text { Porcentaje } \\
\text { válido }\end{array}$ & $\begin{array}{c}\text { Porcentaje } \\
\text { acumulado }\end{array}$ \\
\hline $\begin{array}{l}\text { Suspendido } \\
\text { pagante }\end{array}$ & 6 & 6.7 & 6.7 & 6.7 \\
$\begin{array}{l}\text { permanente } \\
\text { pagante }\end{array}$ & 21 & 23.3 & 23.3 & 30.0 \\
transitorio & 14 & 15.6 & 15.6 & 45.6 \\
normal & 48 & 53.3 & 53.3 & 98.9 \\
$\begin{array}{l}\text { Hijo de } \\
\text { trabajador }\end{array}$ & 1 & 1.1 & 1.1 & 100.0 \\
Total & 90 & 100.0 & 100.0 & \\
\hline
\end{tabular}

\section{Situación de los estudiantes}

La situación de un estudiante, mide su desempeño académico a lo largo de sus estudios universitarios, en este caso podemos observar en el cuadro $\mathrm{N}^{0} 08$, que casi un $29 \%$ de los estudiantes son repitentes, un $51.1 \%$ son de comportamiento académico regular y un $20 \%$ presentan buen desempeño académico, considerando en esta categoría a los que obtuvieron un alto rendimiento y a los invictos.
Cuadro No. 08: Distribución de los estudiantes según su situación

\begin{tabular}{lcccc}
\hline $\begin{array}{l}\text { Situación del } \\
\text { estudiante }\end{array}$ & Frecuencia & Porcentaje & $\begin{array}{c}\text { Porcentaje } \\
\text { válido }\end{array}$ & $\begin{array}{c}\text { Porcentaje } \\
\text { acumulado }\end{array}$ \\
\hline repitente & 26 & 28.9 & 28.9 & 28.9 \\
regular & 46 & 51.1 & 51.1 & 80.0 \\
invicto & 16 & 17.8 & 17.8 & 97.8 \\
$\begin{array}{l}\text { alto } \\
\text { rendimiento }\end{array}$ & 2 & 2.2 & 2.2 & 100.0 \\
Total & 90 & 100.0 & 100.0 & \\
\hline
\end{tabular}

\section{GRADO DE INSTRUCCIÓN DE LOS PADRES}

Se analizó el grado de instrucción de los padres 0 apoderados de los estudiantes de la UNCP, encontrando que el $34,4 \%$ de ellos tienen una educación secundaria, seguido por aquellos que tienen una educación superior $(28,9 \%$ ) y un $23,3 \%$ tienen educación superior técnica, mientras que solo un $12 \%$ tienen estudios primarios o inferiores y uno de ellos manifestó no tener instrucción.

Cuadro No. 09: Distribución de los estudiantes por grado de instrucción de los padres

\begin{tabular}{lcccc}
\hline $\begin{array}{l}\text { G. de I. del } \\
\text { Padre }\end{array}$ & Frecuencia & Porcentaje & $\begin{array}{c}\text { Porcentaje } \\
\text { válido }\end{array}$ & $\begin{array}{c}\text { Porcentaje } \\
\text { acumulado }\end{array}$ \\
\hline Sin instrucción & 1 & 1.1 & 1.1 & 1.1 \\
Primaria & 11 & 12.2 & 12.2 & 13.3 \\
$\quad$ Secundaria & 31 & 34.4 & 34.4 & 47.8 \\
$\begin{array}{c}\text { Superior técnica } \\
\quad \text { Superior }\end{array}$ & 21 & 23.3 & 23.3 & 71.1 \\
universitaria & 26 & 28.9 & 28.9 & 100.0 \\
$\quad$ Total & 90 & 100.0 & 100.0 & \\
\hline
\end{tabular}

\section{INGRESO FAMILIAR}

Se analizó el ingreso familiar de los estudiantes encontrando que el $56,7 \%$ tiene ingresos familiares menores a 1000 soles mensuales, siendo el grupo más representativo los hogares que tienen un ingreso familiar de 500 a 1000 nuevos soles. Ver cuadro $\mathrm{N}^{0} 10$.

Cuadro No. 10: Distribución de los estudiantes por ingreso familiar 


\begin{tabular}{|c|c|c|c|c|}
\hline $\begin{array}{l}\text { Ingreso } \\
\text { familiar }\end{array}$ & Frecuencia & Porcentaje & $\begin{array}{l}\text { Porcentaje } \\
\text { válido }\end{array}$ & $\begin{array}{l}\text { Porcentaje } \\
\text { acumulado }\end{array}$ \\
\hline MENOS DE 500 & 12 & 13.3 & 13.3 & 13.3 \\
\hline DE 500 A 1000 & 39 & 43.3 & 43.3 & 56.7 \\
\hline DE 1050 A 1500 & 22 & 24.4 & 24.4 & 81.1 \\
\hline DE 1550 A 2000 & 11 & 12.2 & 12.2 & 93.3 \\
\hline DE 2050 A 3000 & 5 & 5.6 & 5.6 & 98.9 \\
\hline MAS DE 3000 & 1 & 1.1 & 1.1 & 100.0 \\
\hline Total & 90 & 100.0 & 100.0 & \\
\hline
\end{tabular}

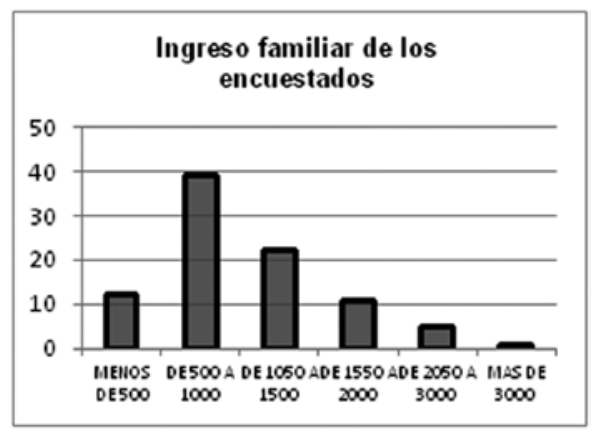

\section{LUGAR DONDE TOMAN SUS ALIMENTOS}

Se analizó el lugar donde los estudiantes toman sus alimentos frecuentemente, encontrando que un $67 \%$ de ellos se alimentan en sus propios hogares y un $13,3 \%$ lo hace en el comedor universitario.

Cuadro No. 14: Distribución de los estudiantes por lugar donde toman sus alimentos

\begin{tabular}{ccccc}
\hline $\begin{array}{c}\text { Tomo mis } \\
\text { alimentos en }\end{array}$ & Frecuencia & Porcentaje & $\begin{array}{c}\text { Porcentaje } \\
\text { válido }\end{array}$ & $\begin{array}{c}\text { Porcentaje } \\
\text { acumulado }\end{array}$ \\
\hline Pensión & 8 & 8.9 & 8.9 & 8.9 \\
$\begin{array}{c}\text { Comedor UNCP } \\
\text { Me preparo la }\end{array}$ & 12 & 13.3 & 13.3 & 22.2 \\
$\begin{array}{c}\text { comida } \\
\text { Otros }\end{array}$ & 5 & 5.6 & 5.6 & 27.8 \\
$\begin{array}{c}\text { Soy de } \\
\text { Huancayo }\end{array}$ & 5 & 5.6 & 5.6 & 33.3 \\
Total & 90 & 66.7 & 66.7 & 100.0 \\
\hline
\end{tabular}

\section{ESTUDIOS DE INGLÉS}

Consideramos que el estudio de Inglés es muy importante para complementar los estudios universitarios, por la dinámica de la ciencia económica, por ello, se estudió si nuestros estudiantes estudian el idioma inglés encontrando que solo el $12 \%$ de ellos estudian Inglés.

Cuadro No. 16: Distribución de los estudiantes por estudios de inglés

\begin{tabular}{ccccc}
\hline $\begin{array}{c}\text { Estudio } \\
\text { Inglés }\end{array}$ & Frecuencia & Porcentaje & $\begin{array}{c}\text { Porcenta } \\
\text { Porcentaje } \\
\text { válido }\end{array}$ & $\begin{array}{c}\text { je } \\
\text { acumula } \\
\text { do }\end{array}$ \\
\hline no & 79 & 87.8 & 87.8 & 87.8 \\
si & 11 & 12.2 & 12.2 & 100.0 \\
Total & 90 & 100.0 & 100.0 & \\
\hline
\end{tabular}

\section{DISCUSIÓN}

Los factores que influyen en el rendimiento académico de los estudiantes de la UNCP, son: modalidad de ingreso; dependencia económica de los estudiantes, y lugar donde toman sus alimentos frecuentemente. Tal como se observa en los cuadros respectivos.

Cuadro $N^{0}$ 16. ANOVA para el rendimiento académico de los estudiantes, según modalidad de ingreso; dependencia económica y lugar donde toman sus alimentos frecuentemente.

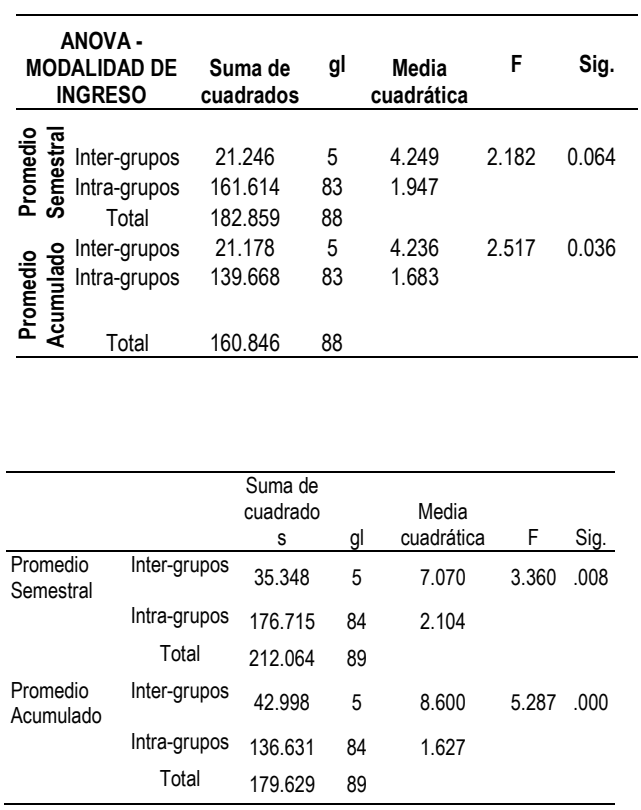




\begin{tabular}{cccccccc}
\hline & & $\begin{array}{c}\text { Suma de } \\
\text { cuadrados }\end{array}$ & gl & $\begin{array}{c}\text { Media } \\
\text { cuadrática }\end{array}$ & F & Sig. \\
\hline & Inter-grupos & 36.808 & 4 & 9.202 & 4.46 & 0 \\
Promedio & & & & & 3 & 3 \\
Semestral & Intra-grupos & 175.255 & 85 & 2.062 & & \\
& Total & 212.064 & 89 & & & \\
& Inter-grupos & & & & & .0 \\
Promedio & & 36.275 & 4 & 9.069 & 5.37 & 0 \\
Acumulado & Intra-grupos & 143.354 & 85 & 1.687 & & \\
& Total & 179.629 & 89 & & & \\
\hline
\end{tabular}

El resultado obtenido puede explicar que estas variables analizadas, si bien al principio ejercen una seria influencia en el comportamiento académico de los estudiantes, sin embargo, a medida que van superando los primeros semestres, el efecto de ellas se va tornando menos perceptible, ello se evidencia en los resultados pues se tomó la información con una muestra de corte transversal que nos ha permitido tomar una información de cómo se comportan esta variables en el momento. Para obtener una mejor referencia sería necesario efectuar un estudio longitudinal para percibir los cambios de manera más puntual.

\section{CONCLUSIONES}

- Se encontró evidencia que las variables modalidad de ingreso, dependencia económica del estudiante, y lugar donde toman sus alimentos, podrían estar influyendo en el rendimiento académico de los estudiantes. Los estudiantes que ingresaron vía traslado externo y primera selección tienen un mejor rendimiento académico, respecto a quienes ingresaron por primeros puestos y traslado interno.

- Las variables, sexo de los estudiantes, semestre académico, Grado de Instrucción de los padres, ocupación de los padres, lugar de origen, tipo de colegio donde estudió, y academia preuniversitaria donde se preparó para el ingreso, no influyen significativamente en el rendimiento académico de los estudiantes.

- Al haber efectuado un estudio de corte transversal, se ha detectado que el efecto de las variables socioeconómicas analizadas se va diluyendo conforme los estudiantes van acumulando más experiencia en las aulas universitarias, por lo que sería muy adecuado efectuar un estudio longitudinal para observar el efecto de esas variables en el rendimiento académico de los estudiantes.

\section{REFERENCIAS BIBLIOGRÁFICAS}

1. Canali Lidia. Fortalezas y debilidades de la docencia universitaria. II encuentro nacional de docentes universitarios católicos. Argentina, 2007.

2. Cano, E. ¿Qué significa evaluar?. Revista Innovación educativa. Universidad de Málaga, 2000.

3. Huaringa, Miguel et al. . La evaluación para el ingreso a la UNCP, .2005.

4. Idrovo, Byron ¿Son las escuelas particulares subvencionadas mejores que las municipales? Estimación de la ecuación de logro escolar para Chile. Tesis Magister. Universidad de Chile, 2007

5. Molnar, Gabriel. Concepto de evaluación aplicada, 2008.

6. Pulido, San Román. Propuesta metodológica para le evaluación de la calidad docente e investigadora. Universidad Autónoma de Madrid, 2003.

7. Quezada, Z. Jenny. Retos en la docencia universitaria del siglo XXI. UNIFE. Centro de Orientación Pedagógica, 2004.

8. Zabal, Antoni, Laia Arnau. Como aprender y enseñar competencias. Tercera edición. Editorial GRAÓ. Barcelona, 2008

Correos electrónicos revisados:

http://educacion.idoneos.com/index.php/118251

www.chasque.net/gamolnar/evaluacion 